\title{
Can Modern Radiotherapy be used for Extensive Skin Field Cancerisation: An Update on Current Treatment Options
}

\author{
Fogarty GB ${ }^{1}$, David Christie ${ }^{2}$, Lynda J Spelman ${ }^{3 *}$, Madeleine J Supranowicz ${ }^{3}$ and Robert J Sinclair ${ }^{3}$ \\ ${ }^{1}$ Genesis Cancer Care, St Vincent's Hospital, Australia \\ ${ }^{2}$ Genesis Cancer Care, Australia \\ ${ }^{3}$ Specialist Connect Services, Australia
}

Received: April 10, 2018; Published: April 25, 2018

*Corresponding author: Lynda J Spelman, Specialist Connect Services, Brisbane, Australia

\section{Abstract}

The use of Radiotherapy (RT) for skin cancer by dermatologists has decreased since the latter half of last century for many reasons. Driven by clinical need, radiation oncologists, radiation biologists and physicists, have progressed RT in many ways over the course of the last fifty years. The creation of multidisciplinary meetings for clinicians involved in skin cancer has put the specialisties of dermatology and radiation oncology in touch. With better modalities and techniques, is there a new role for RT in skin cancer?. A particular scenario is the treatment of Extensive Skin Field Cancerisation (ESFC), where in situ disease can cause significant symptoms and can lead to invasive disease. Current dermatologic and traditional radiation treatments have been disappointing, especially for large convex surfaces of sun-exposed areas such as scalps. These therapies all suffer from a top down problem. To give enough treatment to fully sterilize in situ disease in deep skin appendages, unacceptable side effects can be suffered in the more superficial layers, sometimes leading to a lack of compliance. This review explains recent advances in RT that allow a more homogenous RT dose through the skin treatment volume. Trials need to be performed with modern RT in ESFC. The review also attempts to set some meaningful definitions that can be used for trials. Hopefully these efforts will lead to better oncological, functional, and cosmetic outcomes for patient suffering from ESFC.

Keywords: Skin Cancer; Squamous Cell Carcinoma; Bowens Disease; Radiotherapy; Volumetric Modulated arc Therapy; Review; Field Cancerisation

Abbreviations: RT: Radiotherapy; RCT: Randomised Controlled Trials; ESFC: Extensive Skin Field Cancerisation; IEC: Intraepidermal carcinoma; AK: Actinic Keratosis; EBRT: External Beam Radiotherapy; GTV: Gross Tumour Volume; BT: Brachytherapy; Clinical Target Volume; CTV: Clinical Target Volume

\section{Introduction}

Radiotherapy (RT) for skin cancer was a common treatment administered by dermatologists until the 1980s. Better surgical and topical treatments, coupled with increasing radiation regulatory requirements, led to a decline in the use of RT by dermatologists. RT has continued to evolve in the treatment of other cancers. Driven by radiation oncologists, radiation biologists and physicists, RT has progressed in many ways. High quality Randomised Controlled Trials (RCT) has led to an increase in the indications for RT in many tumour types. The creation of multidisciplinary meetings for clinicians involved in skin cancer has put the specialities of dermatology and radiation oncology in touch. This has led to dermatologists asking whether there is a new role for modern RT in the treatment of skin cancer. A particular scenario is the treatment of Extensive Skin Field Cancerisation (ESFC). Patients can suffer with ESFC caused by chronic ultraviolet radiation exposure (Figure $1)$.

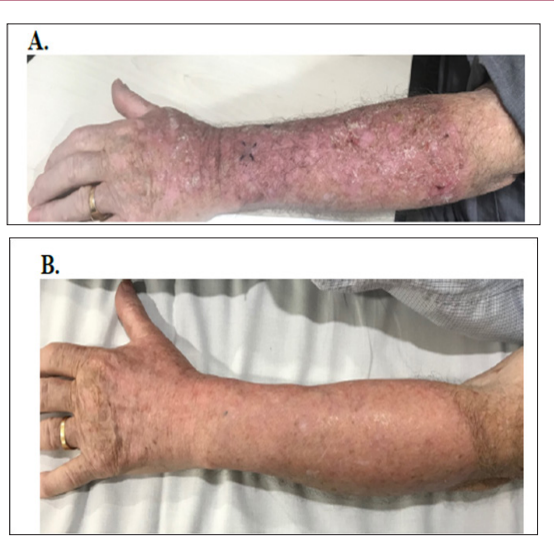

Figure 1: A: ESFC on arms in sun-exposed areas. $B$ : Following modern radiotherapy.

Source: Provided by G Fogarty for this article. 
Actinic Keratosis (AK), Bowen's disease or Intraepidermal carcinoma (IEC) are found in ESFC from which new invasive Cutaneous Squamous Cell Carcinoma (cSCC) can arise[1]. This disease can cause significant morbidity and poor quality of life, with itch, flaking skin and poor cosmesis. Patients often have comorbidities that preclude other treatments, especially surgery if complex closure is required. Patients may also decline surgery because of fear of a poor functional or cosmetic outcome with the tissue loss that surgery entails. Current dermatologic treatments have been disappointing, especially for larger convex surfaces of sun-exposed areas such as scalps. Recurrence at twelve months is common [2-4]. Not all therapies are readily available. Application can be painful. Skin reaction, sometimes a necessary measure of efficacy, can be unsightly, painful and require significant care including dressings.

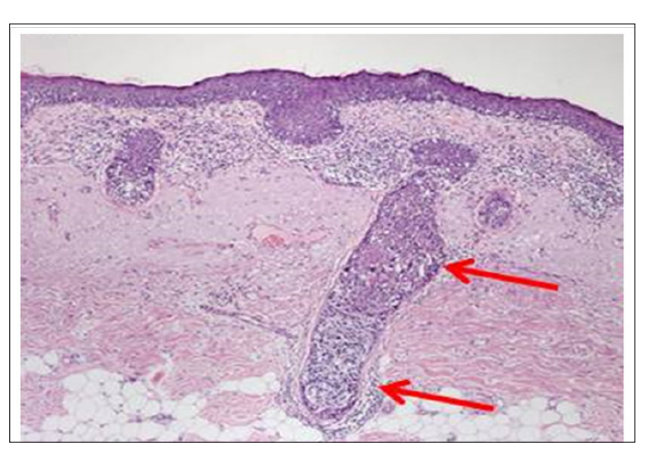

Figure 2: Photomicrograph showing a cross section of cancerous skin with H\&E stain. Skin appendages shown by the red arrows to indicate the depth that must be treated. Lack of treatment penetration to the depth of the appendages may be the cause of future in-field field recurrences. Therapies with a top down problem will not sterilise the adnexal structures without potential unacceptable side effects being suffered in the more superficial layers-see text.

Source: Figure provided courtesy of Prof Richard Scolyer.

Compliance can be difficult if application depends on the patient [5]. Cryotherapy can cause hypopigmentation and scarring. These therapies all suffer from a similar problem. The deepest appendages on male scalp have been shown to be $4.5 \mathrm{~mm}$ depicted in Figure 2[6]. To deliver enough treatment to fully sterilize in-situ disease deep in skin appendages, unacceptable side effects can occur in the more superficial layers, leading to a lack of compliance and cure. This review describes recent advances in RT that allow a more homogenous RT dose through the target treatment volume. RT trials with these advances in ESFC are now on going and will hopefully lead to better oncological, functional, and cosmetic results.

\section{Traditional RT Modalities also suffer from the Top downProblem}

Traditional modalities of radiotherapy treatment can be broadly divided into two types. Teletherapy or External Beam Radiotherapy (EBRT), involves radiation created outside the patient and beamed into the target from a source relatively distant. Brachytherapy (BT) involves the use of radioactive sources that are laid onto or into the target. BT for skin (Figure 3) is delivered via a specially constructed surface mould. The homogeneity of the surface dose depends on the distance of the sources from each other, and the distance of the skin to the sources (called "standoff"). Surface moulds contain catheters along which a high dose rate source travels under computer control. The energy of the radiation emitted from the source and the source standoff distance will also determine the dose at depth in the skin. Increasing the thickness of standoff will create a more homogenous dose in the skin but will increase significantly the time taken to deliver treatment. Increasing the standoff thickness will also increase dose to deeper structures [7]. This is a top down problem. The top down problem implies that the therapeutic effect of the treatment is focused at the epidermis and decreases in efficacy with depth.

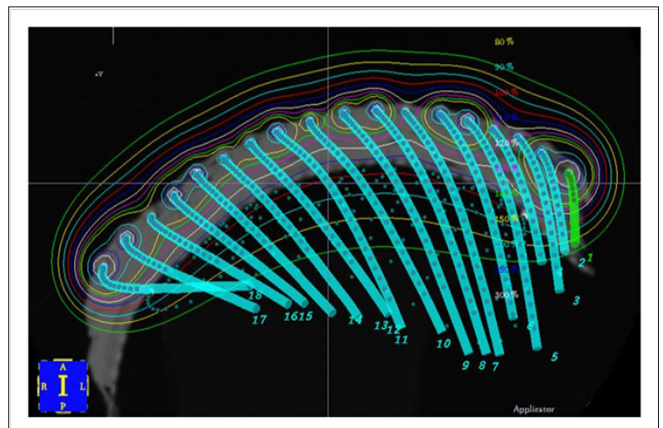

Figure 3: A representation of a brachytherapy mould where the brachytherapy catheters are kept in a pattern by the mould, usually separated by equal spacing. The mould is made of a water-equivalent material called bolus or build up. Bolus between the brachytherapy catheters and the skin that attenuates the radiation. The thickness of this bolus is called the standoff distance. This enables the dose cloud, by the time it gets to the skin, to have a homogeneous wave front. See the thin red line.

Source: Provided by G Fogarty for this article.

Traditional modalities of EBRT for skin cancer have included beams of either photons or electrons. EBRT comes from a point source (Figure 4). A machine that has a stationary point source is ideal for treating small areas of the skin close to sensitive structures, especially areas concave to the beam front (e.g. inner canthus, nasal-alar groove) of Figure4. Superficial radiotherapy (SXRT) is ideal in treating a lesion that is concave to the beam e.g. inner canthus, nasal-alar groove, with little exposure of underlying organs at risk. However, the short SXRT SSD means the field size is limited to $8 \mathrm{~cm}$ at skin surface so SXRT can only effectively treat lesions less than $6 \mathrm{~cm}$ in diameter. SXRT was the treatment often delivered by dermatologists. EBRT beams can consist of either photons or electrons; both are generated by linear accelerators using megavoltage energies. The advantage of electrons over SXRT is that electrons can treat a greater area of skin. Electrons have a better depth dose curve meaning that the top down problem is not as great. There are however several disadvantages with electrons. Electrons need a layer of tissue-equivalent material called bolus to bring the maximum dose onto the skin. The use of bolus increases the set-up time and the uncertainty of treatment. Electron beams have a wider penumbra and so need a bigger field, meaning more normal tissue will be irradiated. 


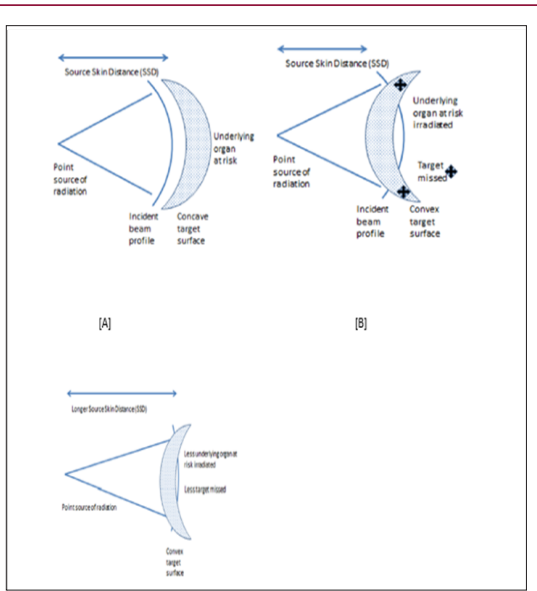

Figure 4: A: This cartoon shows schematically a fixed point source of radiation with a short sourceto-surface distance (SSD) e.g. a superficial X ray machine (SXRT).

B: For lesions that are convex tothe beam, however, there can be significant under dosing of the target leading to treatment failure atthe periphery and overdosing of deeper organs, increasing the risk of side effects.

C: This is anothertop down problem. Compromise can include using machines with a longer SSD so that the beamprofile is flatter when incident on the lesion. A machine with a longer SSD than an SXRT machine isthe linear accelerator, which can emit electrons. Electrons are ideal irradiating larger fields of skinto a more homogeneous dose. Electrons, however are complex. Source: Provided by G Fogarty for this article.

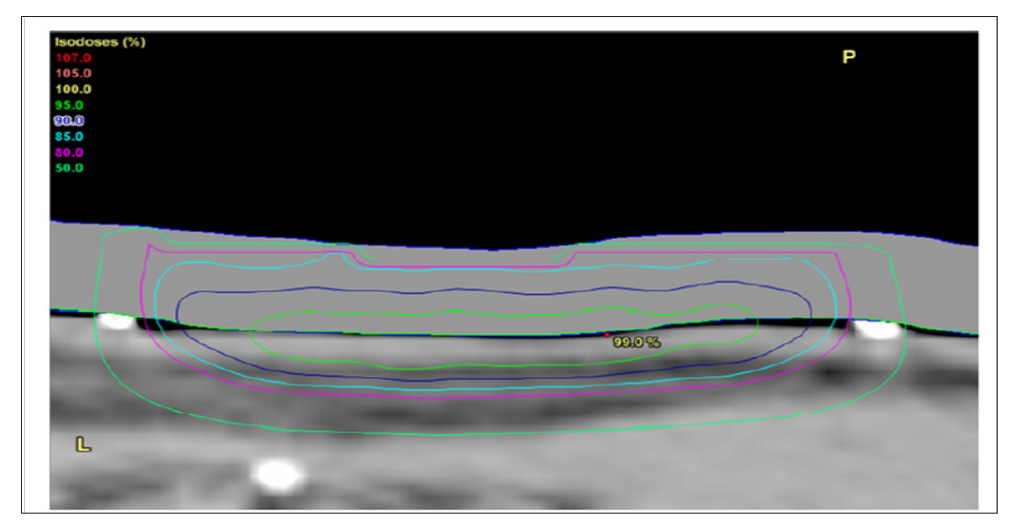

Figure 5: Electron beams are most useful when a flat surface is treated with a beam perpendicular to the surface. In this diagram the $90 \%$ isodose line (green), which is the line that used to calculate the prescription, straddles the skin surface. Note the wires are marking the field. One can see a significant dose drop-off towards the subcutis, meaning less subcutaneous dose, an advantage of electrons.

Source: Provided by G Fogarty for this article.

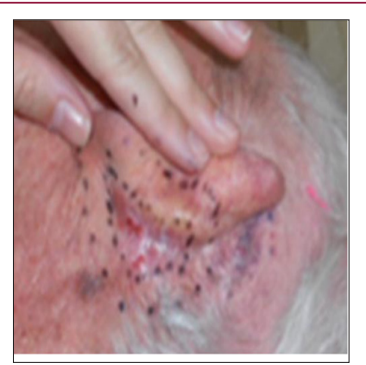

Figure 6: Electron beams are more useful if there is a flat field. The skin surface can be modified to make it flat. For example, one can tape the ears forward in order to treat the posteromedial surface of the pinna. Note the inner dotted area which denotes GTV and the outer dotted area denotes the planning Target Volume (PTV). The PTV is larger for electron beams than for SXRT as electrons have a wider penumbra.

Source: Provided by G Fogarty for this article
Electron beams are best treating a flat field that is perpendicular to the direction of the beam (Figure 5). The skin that is to be treated can be modified to make it flat. For example, noses can be taped flat to one side when treating the nasal ala. One can tape the ears forward to treat the postero-medial surface of the pinna (Figure 6). One can also use bolus material to make the field as flat as possible, for example, filling a conchal bowl. Electron dosimetry can be difficult when treating in homogeneities, for example, the nose with air tissue interfaces such as in the nostril. The treatment staffs need to pack the nostril so it absorbs dose in the same way as a solid organ. This packing takes time, is uncomfortable especially towards the end of treatment when side effects like mucositis are common and decreases reproducibility of the set-up from day to day.To treat larger skin areas, several electron fields may need to be junctioned together. The dose of radiation in the junction areas between the fields can be difficult to measure (Figure 7), which can result in under-dosing tumour or overdosing normal tissue, with 
the problems of either recurrence of the cancer or late normal tissue effects.

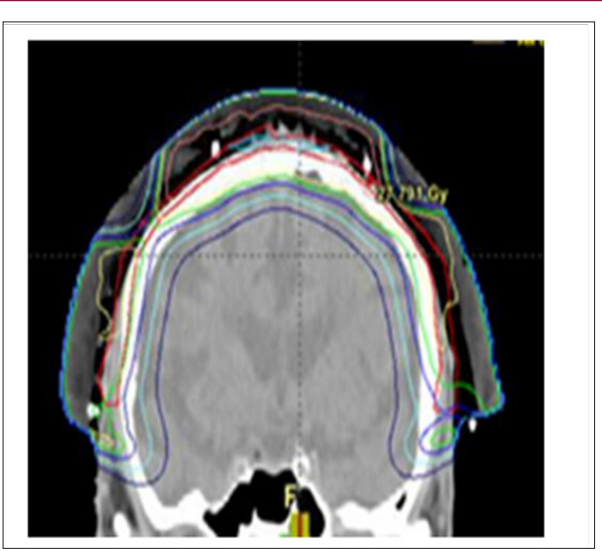

Figure 7: A sagittal CT planning image showing treatment of the scalp with electron fields junctioned together. Note the inferior dosimetry at the field junctions. This plan is not acceptable.

Source: Figure provided courtesy of Prof Peter Graham.

Overall, traditional RT modalities fall short in treating large fields of skin cancer in two major ways.

a) The dose homogeneity is not uniform through the thickness of the target. There is too much dose to the surface and too little to the depth of the appendages. This is the same top down problem as traditional dermatological treatments have. Solutions to this have involved unacceptable increases in dose to deeper tissues.

b) The fields are small due to technical reasons, leading to the need for junctioning of radiation fields and the consequent inaccuracies of dose at the junctions.

\section{Recent Advances in RT}

RT of skin cancer has benefitted from significant recent advances in general RT.

These include:

a) Better understanding of the target to be treated, and normal tissues to be spared

b) Better modalities of radiotherapy planning, delivery and verification

c) Improved techniques in using these modalities; and

d) Better understanding of the total dose needed and adequate fractionation.

\section{Better Understanding of the RT Target to be Treated}

Traditionally RT treatments were defined in terms of radiation fields. However, cancer, and normal tissues to be spared, occurs in volumes. The definition of volumes for use in radiation oncology is outlined in a consensus document, ICRU 50 [8]. These concepts have been helpful in modern radiotherapy and are defined briefly below: Gross Tumour Volume (GTV) is macroscopic cancer defined clinically. Physical Examination (PE) is aided by the use of imaging.
In skin, imaging can include photography, dermoscopy and Reflectance Confocal Microscopy (RCM). Targeted biopsy is another aid to PE. Clinical Target Volume (CTV) is the volume that harbours microscopic tumour. The CTV must be adequately treated to achieve cure. The target in large fields of skin cancer that needs treatment is the volume that is involved with a concretization process. In skin, this volume is the product of the area on skin multiplied by a depth including epidermis and the deepest skin appendages. This distance is $5 \mathrm{~mm}$ from the skin surface, or until an oncological boundary is reached e.g. the skull.

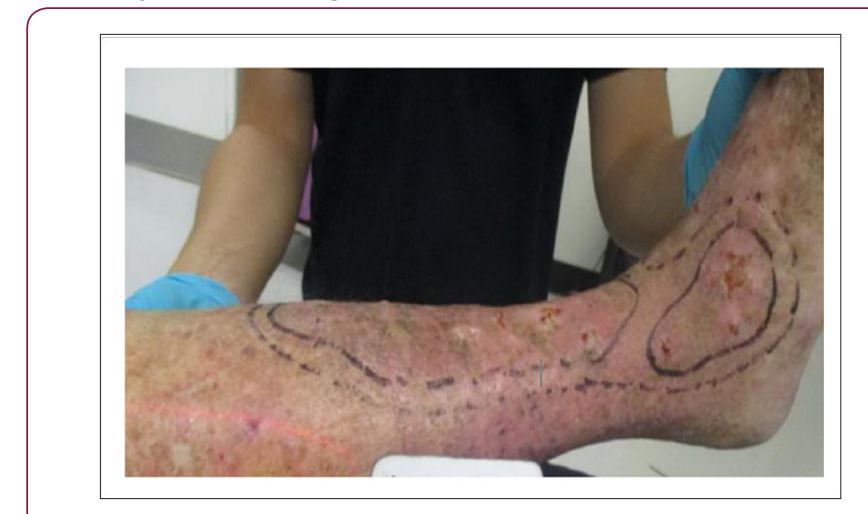

Figure 8: Treatment volumes are determined by marking areas on skin at the planning phase and multiplying by the depth. In this example of skin field concretization on a leg. The CTV is the inner marking. The PTV in this case is a one-centimetre radial expansion on the CTV. All the PTV will receive therapeutic dose.

Source: Provided by G Fogarty for this article.

Planning target volume (PTV) is a further expansion to account for variation in daily treatment variables. PTV depends on the effectiveness of patient immobilization protocols. The PTV size also depends on the modality of treatment used, which will determine the size of the penumbra. PTV has been gradually reducing over years with better and more reproducible immobilization techniques, daily image guided radiotherapy and the ability to contour the targets on the planning Computed Tomography (CT). Organs at Risk (OARs) are normal tissues within the RT entrance or exit beam that are sensitive to RT. Contouring these on a planning CT allows measurement of the radiation received by that organ so that the dose can be well within that associated with side effects. These tolerance doses have been calculated over years of experience and are well known in the radiotherapy world (Figure 8).

\section{Better modalities of radiotherapy planning, delivery and verification}

Better modalities of radiotherapy delivery include the use of megavoltage photon beams produced by a Linear Accelerator (or "linac").

These advances include:

a) The use of megavoltage photon beams (MVT). MVT has a longer SSD over SXRT, meaning that the incident radiation front is almost parallel to skin. (Figure 4C).

b) The use of computer tomography (CT) planning scans. MVT enables the use of computer tomography (CT) planning 
scans taken in the treatment position, and planning systems. The volumes to be treated and avoided are contoured by the radiation oncologist on these scans. Doses are prescribed for each volume. Dose Volume Histograms (DVHs) can be constructed and doses per volume on each individual patient plan can be compared to standardized doses for CTV and PTV and OARs. These standardized doses are known to predict cure for tumour bearing volumes and are associated with acceptable side effects in normal tissue volumes. Technology with the planning system is then used to optimize the plan to achieve these bench marks, ensuring tumour cure and normal tissue tolerance at the end of treatment (Figure 9).
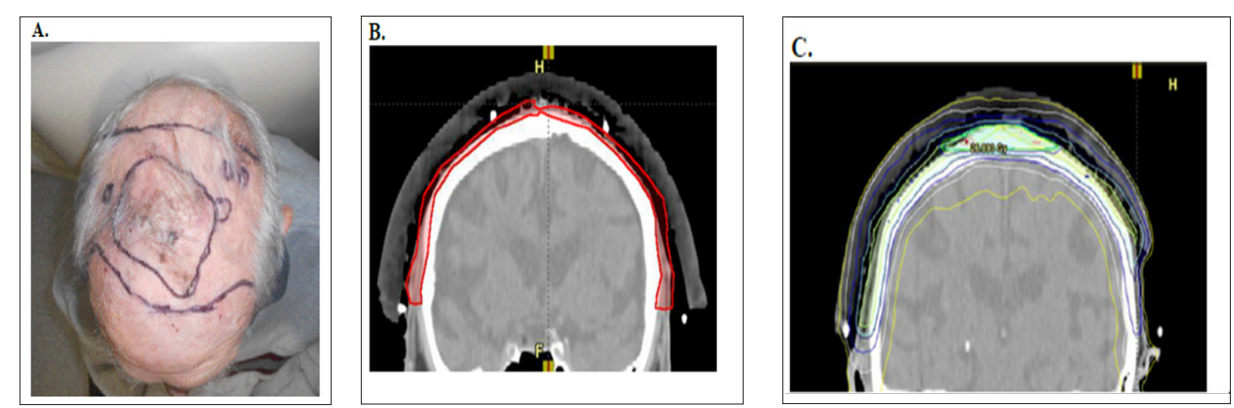

Figure 9: Photographs and CT plans of a patient preparing for VMAT.

A: Mark up of a scalp field at planning showing areas of gross tumour within the area of ESFC.

$\mathrm{B}$ : CTV is contoured on the planning scan. Red volume represents ESFC.

C: The resultant dosimetry avoiding brain. The dose homogeneity is uniform throughout the target. The dose profile across the field is uniform with no need for junctioning radiation fields. Green volume represents gross tumour.

c) The use of Intensity modulated radiotherapy (IMRT). IMRT, using automated multileaf collimators in the head of the linac, enables the intensity of the beam to be modified during beam-on time, sculpting the dose to the contoured volumes. Prior to this advance radiotherapy was initially Two Dimensional (2D) and based on fields. The application of CT technology in planning accelerated the uptake of progression to Three Dimensional Conformal Radiotherapy (3DCRT) with the concept of volume treatment. However, RT still came in "blocks". IMRT allowedtreatment around curves, especially sparing dose to OARs in the concavity of a volume requiring treatment (e.g. brain underneath a scalp)

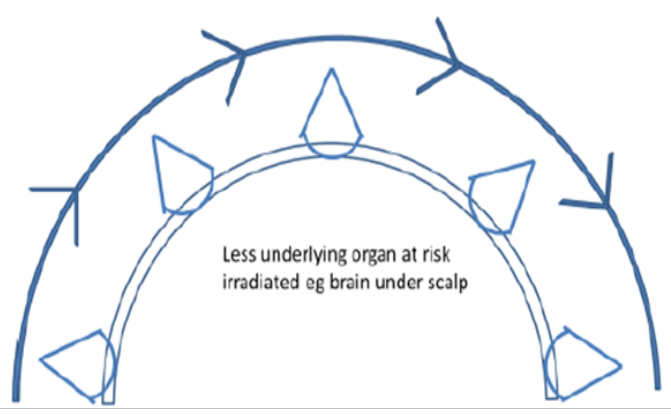

Figure 10: Cartoon showing that VMAT allows the point source of radiation to move around convex surface in an arc resulting in greater conformality.

Source: Provided by G Fogarty for this article.

d) The use of Volumetric modulated arc therapy (VMAT). VMAT, the latest evolution of IMRT, is essentially the application of CT technology to IMRT treatment. The point source is now in motion and not fixed as in traditional EBRT modalities. The patient can be continuously irradiated with the RT source in motion rather than only from fixed angles (Figure 10). e) Vary the dose rate during beam on time. Modern linacs can also vary the rate at which the dose is delivered as the gantry rotates around the patient, further enabling dose conformality;

f) Use of CT planning, MVT and VMAT enable dose homogeneity throughout the volume. The top down problem has been overcome using these modalities;

g) Use of Image Guided RT (IGRT). MVT photon beams can penetrate through the body to expose an X-ray detector on the opposite side of the body. This allows for daily quality assurance imaging during beam on time to verify that the intended volume has been irradiated. This is called image guided radiotherapy. The assurance that the correct volumes are treated, only achievable by daily imaging, means that the PTV can be significantly decreased, thereby sparing more normal tissue from radiation. The traditional EBRT modalities were not capable of this; and

h) Rapid delivery. VMAT can be delivered rapidly, meaning that the patients are immobile on the treatment bed for a short time. Time in the treatment room is the scarce resource in the radiotherapy department. Time in the room for VMAT averages approximately 10 minutes, while for IMRT treatments is 30 minutes. This means that VMAT treatments can be delivered to more patients, rather than being rationed, as the initial IMRT cases were. VMAT is therefore becoming the standard technique used in many modern departments.

\section{Improved Techniques in Using These Modalities}

The significance of these advantages for skin cancer is that radiotherapy can now treat large curved convex surfaces, such as those found in ESFC. Large convex surfaces of ESFC can be found covering anatomical sites such as the scalp, forehead, cheeks, forearms, legs, chest, upper back, and shoulders, VMAT can now 
be used to treat these skin surfaces with definitive MVT photon RT (Figure 9). These advances have overcome the problems of traditional RT. The dose can now be more uniform throughout the target, with no need for junctioning radiation fields.

\section{Better understanding of the total dose needed and adequate fractionation}
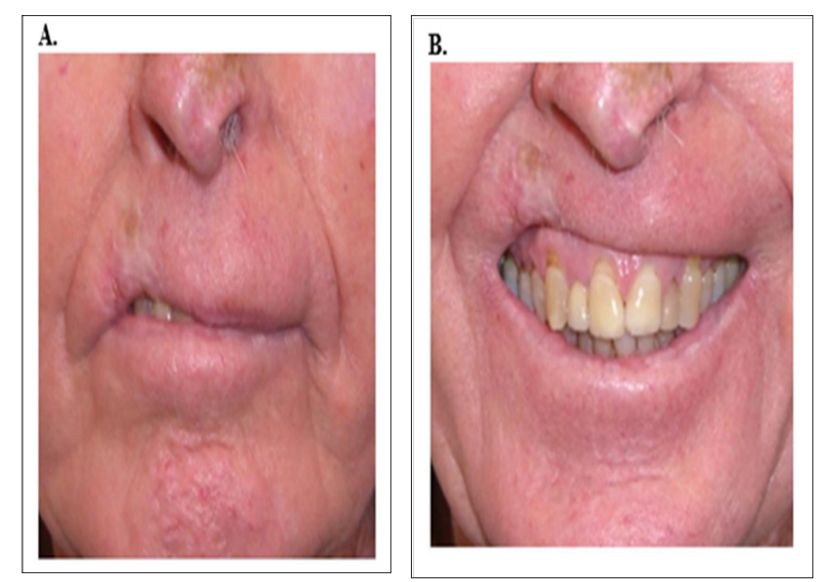

Figure 11: A: cSCC lip treated years ago with hypofractionation leading to telangiectasia, cicatrisation, atrophy and hypopigmentation.

B: Hypofractionation has had a deleterious cosmetic effect on this person's smile

Source: Provided by G Fogarty for this article

Table 1: Table of biologically equivalent dose (BED) doses for microscopic disease for ESFC therapy.

\begin{tabular}{|c|c|c|c|c|c|c|}
\hline $\begin{array}{c}\text { Total } \\
\text { dose } \\
\text { (Gy) }\end{array}$ & $\begin{array}{c}\text { No of } \\
\text { fractions } \\
\text { /weeks of } \\
\mathbf{R T}\end{array}$ & $\begin{array}{c}\text { Dose } \\
\text { per } \\
\text { fraction }\end{array}$ & $\begin{array}{c}\text { BED* } \\
\text { early }\end{array}$ & $\begin{array}{c}\text { BED** } \\
\text { late }\end{array}$ & $\begin{array}{c}\text { EQD2*** } \\
\boldsymbol{\alpha} / \boldsymbol{\beta} \\
\mathbf{= 1 0}(\mathbf{G y})\end{array}$ & $\begin{array}{c}\text { EQD2**** } \\
\boldsymbol{\alpha} / \boldsymbol{\beta} \\
\mathbf{3} \text { (Gy) }\end{array}$ \\
\hline 60 & $30 / 6$ & 2 & 72 & 100 & 60 & 60 \\
\hline 55 & $25 / 5$ & 2.25 & 68.91 & 98.44 & 56.15 & 57.75 \\
\hline 50 & $20 / 4$ & 2.5 & 62.5 & 91.67 & 52.08 & 55 \\
\hline 45 & $15 / 3$ & 3 & 58.5 & 90 & 48.75 & 54 \\
\hline
\end{tabular}

Source: (Fowler JF1989, Fowler JF 2010)

Recent contributions to this aspect of RT include the importance of understanding the total dose and fractionation to achieve better oncological, functional, and cosmetic results. The total dose needed to sterilise skin field concretization is intuited from head and neck squamous cell carcinoma [9] which requires at least 60 Gy given in standard fractionation. The total dose is given in many smaller doses or fractions to maximise normal tissue repair between the fractions. Standard fractionation is 2 Gy per day. 60 Gy in 2 Gy fractions means six weeks of treatment. To attend 6 weeks of five-days-a-week treatment may be inconvenient. Alternative fractionation patterns can be devised using mathematical formulae [10,11](Table 1). However, at fraction sizes greater than 2.5 Gy per day, late functional and cosmetic results may decline. This is because, at larger fraction sizes, the radiation repair capacity of normal cells is swamped and normal cells die and is eventually replaced by fibrous tissue months to years following treatment. Late radiation side effects such as hypopigmentation, telangiectasia and fibrosis can develop (Figure 11). These problems have prompted dermatologists to seek other treatment solutions in the past. For patients unable to have standard fractionation, compromise fractionation schedules have been developed [12]. Skin cancer especially in the elderly may be more radiosensitive and fewer doses may be needed [13]

\section{Towards Trials of Modern Radiotherapy in ESFC}

Theoretically modern RT may be of benefit in this clinical scenario. To create meaningful studies the scope of the problem needs definition and we would like to propose the following.

\section{Is it ethical to treat patients with ESFC with RT?}

RT has been used for ESFC in the past with good effect. Dinehart, Graham, and Maners [14] treated 16 patients with threedimensional conformal megavoltage radiotherapy (3DCRT) with 40 to 60 Gray (Gy) with complete response in 13 after four weeks of follow-up. Of 10 evaluable patients at one year, nine had continuing complete response. Pipitone and Gloster [15] treated one patent with extensive scalp recurrence with 3DCRT, 60 Gy in 30 fractions, with enduring complete response at 18 months. Barta, Gräfe, and Wollina [16] treated a 66-year-old with a $10 \times 8 \mathrm{~cm}$ field on the scalp full of AK with 28 Gy, given as 4 Gy fractions twice weekly, with a continuing complete response at 14 months. There has been one report so far of using VMAT for skin field treatment [17]. RT traditionally has not been regarded as a first line treatment for ESFC but as a salvage treatment.

\section{Defining ESFC}

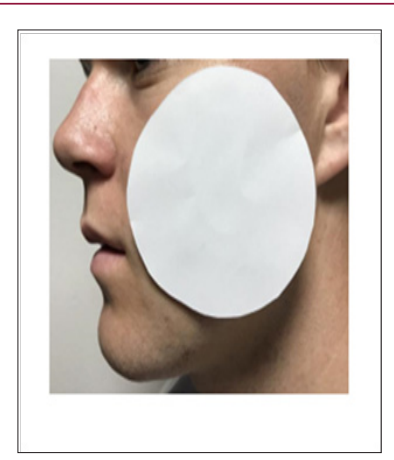

Figure 12: Sizes of a $50 \mathrm{~cm} 2$ field on a cheek of an adult male with an $8 \mathrm{~cm}$ diameter circle.

Source: Provided by G Fogarty for this article

There are various definitions of ESFC. Hofbauer et al. [18] published The Swiss Registry of Actinic Keratosis Treatment (REAKT) Working Group which suggests the presence of two or more AK lesions along with photodamage. Figueras Nart et al.[19] propose a clinical definition, based on an anatomical region having telangiectasia, atrophy, pigmentation and a sandpaper texture. Surgery has been used for ESFC [19]. What is needed for trials is a minimum ESFC size. ESFC can be defined when it is too large for simpler surgical procedures e.g. primary closure, pedunculated flap or skin graft. Complex surgery may then be needed but may not be possible due to patient factors e.g. comorbidities, expectations, prior therapies and patient refusal. We propose for trial purposes 
that ESFC should be beyond the limits of reasonable surgery. Our experience is that a field of $50 \mathrm{~cm} 2$ anywhere on the body is a reasonable definition of ESFC. Examples can be seen Figure 12 an $8 \mathrm{~cm}$ diameter circle. Areas of treatment can also be defined as anatomical regions. These should be treated as unit to ensure after RT that functions and cosmesis is maximized. Irradiating less than these areas can result in unnatural visible field treatment borders (Figure 13).

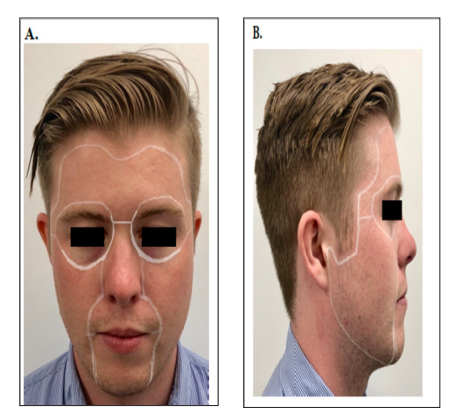

Figure 13: Anatomical regions of the face and neck for RT treatment for ESFC.

A: Anterior projection showing forehead and nasal regions.

B: Lateral view showing forehead, nasal and cheek regions. Source: Provided by R Sinclair and L Spelman for this article

\section{Conclusion}

RT has improved over the last few decades. These improvements have been in better understanding of the target to be treated, and normal tissues to be spared; better modalities of radiotherapy planning, delivery and verification; improved techniques in using these modalities in skin cancer; and better understanding of the total dose needed and adequate fractionation. RT may now have a role in areas of dermatology that have defied durable treatments including ESFC. Current dermatologic and traditional radiation treatments have been disappointing. These therapies all suffer from a top down problem. To give enough treatment to fully sterilize in situ disease in deep skin appendages, unacceptable side effects can be suffered in the more superficial layers, sometimes leading to a lack of compliance.Trials are now needed to place modern RT among the treatments usually given for this condition. We set the scene for doing trials by defining what constitutes ESFC as areas of skin $50 \mathrm{~cm} 2$ and over, in anatomical regions. If radiation oncologists and dermatologists cooperate, trials testing RT for the treatment of ESFC should be possible and will lead to better and more durable oncological, functional, and cosmetic outcomes for patients suffering from ESFC.

\section{Acknowledgement}

The authors wish to acknowledge the contirubiton of figures: Figure 2 provided courtesy of Prof Richard Scolyer and Figures 7 and Figure 9C: provided courtesy of Prof Peter Graham. Also to Kristy Frappell for administrative support.

\section{References}

1. Green AC (2015) Epidemiology of actinic keratoses Actinic Keratosis. Karger Publishers (46): 1-7.
2. Askew DA, Mickan SM, Soyer HP, Wilkinson D (2009) Effectiveness of 5- fluorouracil treatment for actinic keratosis-a systematic review of randomized controlled trials. International journal of dermatology 48(5): 453-463.

3. Gupta AK, Paquet M, Villanueva E, Brintnell W (2012) Interventions for actinic keratoses. Cochrane Database Syst Rev 12.

4. Krawtchenko N , Roewert Huber J, Ulrich M, Mann I, Sterry W, et al. (2007) A randomised study of topical 5\% imiquimod vs. topical 5fluorouracil vs. cryosurgery in immunocompetent patients with actinic keratoses: a comparison of clinical and histological outcomes including 1-year follow-up. British Journal of Dermatology 157(s2): 34-40.

5. Philipp Dormston WG (2015) Field cancerization: from molecular basis to selective field-directed management of actinic keratosis Actinic Keratosis. Curr Probl Dermatol (46): 115-121.

6. Fogarty G, Hong A, Scolyer R, Lin E, Haydu L, et.al (2014) Radiotherapy for lentigo maligna: a literature review and recommendations for treatment. British Journal of Dermatology 170(1): 52-58.

7. Santos DE, Green JA, Bhandari N, Fogarty B, Hong A, et al. (2015) Tangential Volumetric odulated Radiotherapy-A New Technique for Large Scalp Lesions with a Case Study in Lentigo Maligna. International Journal 19(2): 223-236.

8. Rosenthal DI, Mohamed AS, Garden AS, Morrison WH, El Naggar AK, et al. (2017) Final report of a prospective randomized trial to evaluate the dose-response relationship for postoperative radiation therapy and pathologic risk groups in patients with head and neck cancer. International Journal of Radiation Oncology Biology Physics, 98(5): 1002-1011.

9. Fowler JF (1989) The linear-quadratic formula and progress in fractionated radiotherapy. The British journal of radiology 62(740): 679-694.

10. Fowler JF (2010) 21 years of biologically effective dose. The British journal of radiology 83(991): 554-568.

11. Carney G, Fogarty G, Moutrie Z , Rose McLaren K (2017) Adaptive Split Course Radiotherapy for Skin Cancers Decreases Toxicity and Improves Compliance 2(1): 00015

12. Wambersie A, Landberg T (1999) ICRU Report 62: Prescribing, Recording and Reporting Photon Beam Therapy (Supplement to ICRU Report 50) International Commission on Radiation Units \& Measurements 32(1).

13. Dinehart SM, Graham M, Maners A (2011) Radiation therapy for widespread actinic keratoses. The Journal of clinical and aesthetic dermatology 4(7): 47.

14. Pipitone MA, Gloster HM (2006) Superficial squamous cell carcinomas and extensive actinic keratoses of the scalp treated with radiation therapy. Dermatologic surgery 32(5): 756-759.

15. Bart U, Gräfe T, Wollina U (2000) Radiation therapy for extensive actinic keratosis. Journal of the European Academy of Dermatology and Venereology 14(4): 293-295.

16. Veness M (2017) Hypofractionated radiotherapy in older patients with non-melanoma skin cancer: Less is better. Australas J Dermatol.

17. Hofbauer G, Anliker M, Boehncke WH, Brand C, Braun R, et al. (2014) Swiss clinical practice guidelines on field cancerization of the skin. Swiss Med Wkly 144: w14026.

18. Figueras Nart I, Cerio R, Dirschka T, Dréno B, Lear J, et al. (2017) Defining the actinic keratosis field: a literature review and discussion. Journal of the European Academy of Dermatology and Venereology 32(4): 544563.

19. Desai SC, Sand JP, Sharon JD, Branham G, Nussenbaum B (2015) Scalp reconstruction: an algorithmic approach and systematic review. JAMA facial plastic surgery 17(1): 56-66. 
(c) (i) This work is licensed under Creative

Submission Link: https://biomedres.us/submit-manuscript.php

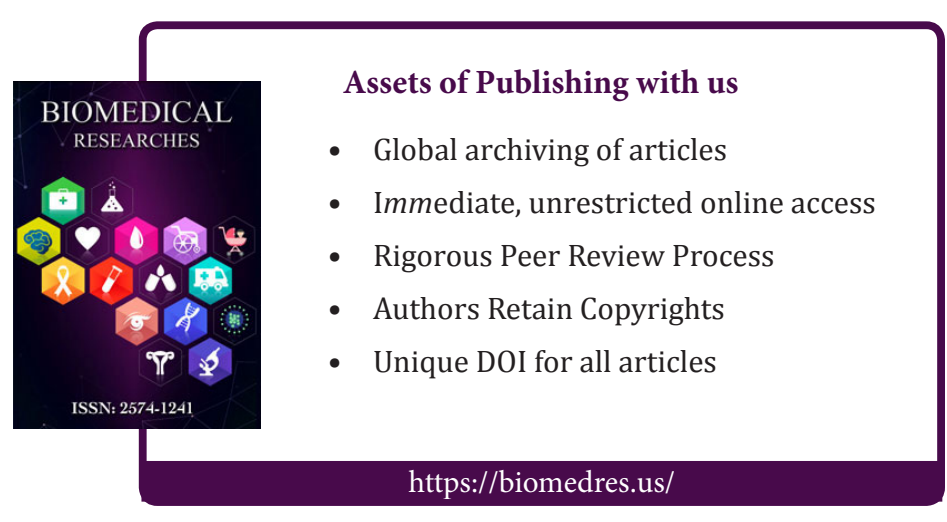

